Radiologe 2022 ·62:140-148

https://doi.org/10.1007/s00117-021-00954-9

Angenommen: 9. Dezember 2021

Online publiziert: 18. Januar 2022

(c) The Author(s), under exclusive licence to Springer Medizin Verlag GmbH, ein Teil von Springer Nature 2022

\section{Röntgenuntersuchungen des Thorax bei Kindern und Jugendlichen}

\author{
Indikationen und Limitationen
}

Zusammenfassung

Klinisches Problem: Die Röntgenaufnahme des Thorax ist die häufigste konventionelle Röntgenuntersuchung im Kindes- und Jugendalter. Das Ziel dieser Übersichtsarbeit ist es, den Benefit der Röntgenmodalität, aber auch ihre Limitationen darzulegen. Methode: Neugeborene erhalten verglichen mit älteren Kindern proportional die häufigsten Röntgenaufnahmen des Thorax. Nach der Neugeborenenperiode setzt diese Übersichtsarbeit einen Fokus auf die Diagnostik entzündlicher Lungenveränderungen, die Fremdkörperaspiration, die Detektion von Rundherden und die zystische Fibrose.

Methodische Innovationen: Aufgrund verbesserter Technologien sinkt die Strahlenexposition konventioneller Thoraxaufnahmen kontinuierlich. Jedoch werden die anderen Bildgebungsmodalitäten ebenfalls stetig optimiert, so dass insbesondere die strahlungsfreien Alternativen Magnetresonanztomographie (MRT) und Sonographie bei Vorliegen einer bestimmten klinischen Fragestellung in Erwägung gezogen werden sollen.

Empfehlung für die Praxis: Auch wenn die diagnostische Aussagekraft von RöntgenThorax-Aufnahmen häufig geringer als von Computertomographie (CT) oder MRT ist, hat sie weiterhin aufgrund ihrer ubiquitären Verfügbarkeit und der relativ einfachen Durchführbarkeit einen hohen Stellenwert im Kindes- und Jugendalter.

\title{
Schlüsselwörter
}

Neonatalperiode · Pneumonie · Zystische Fibrose · Computertomographie · Magnetresonanztomographie

\section{Hintergrund}

Auch im 21. Jahrhundert ist die Röntgenuntersuchung des Thorax ein Standardverfahren im Kindes- und Jugendalter. Die Projektionsradiographie des Thorax stellt die häufigste pädiatrische konventionelle Röntgenuntersuchung dar [1]. Ein wesentlicher Vorteil von Röntgen-ThoraxUntersuchungen ist die relativ schnelle Durchführbarkeit mit der Möglichkeit der Akquisition als Bettaufnahmen auch auf In- tensivstationen. Aufgrund ihrer Häufigkeit tragen Röntgen-Thorax-Untersuchungen trotz der relativ geringen Strahlendosis der einzelnen Aufnahmen zu der kollektiven und kumulativen Strahlenexposition wesentlich bei [1]. Durch verbesserte Technologien sinkt die Strahlenexposition konventioneller Aufnahmen kontinuierlich. Nach einer Studie von Seidenbusch und Schneider [2] konnte der Dosisbedarf von Radiographien des Thorax bei pädiatrischen Patienten innerhalb von 

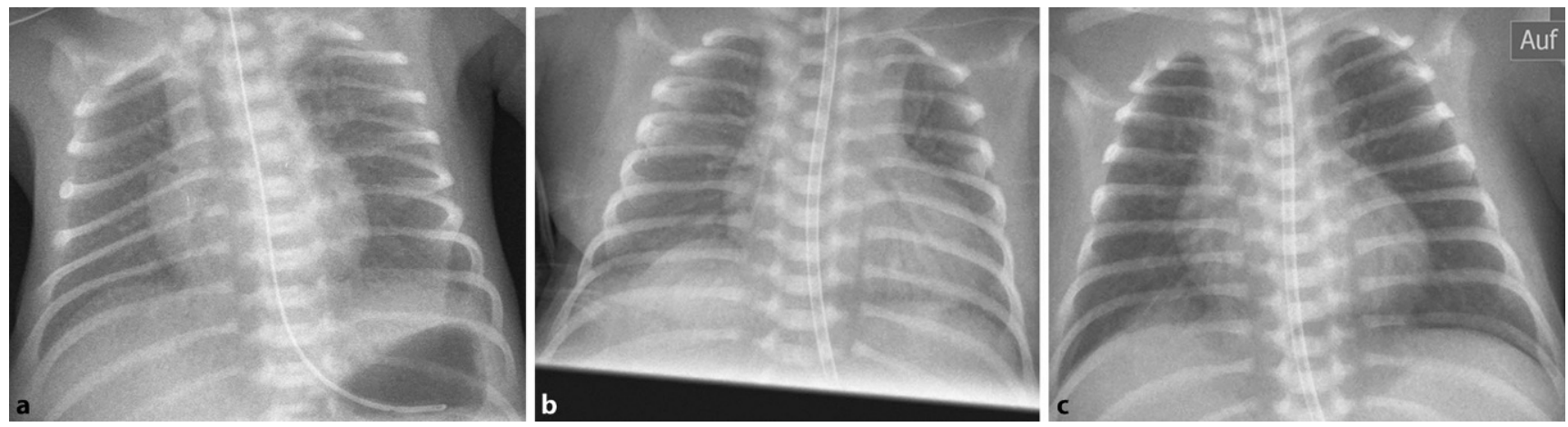

Abb. $1 \Delta$ a Röntgenaufnahme des Thorax eines Frühgeborenen der 28. Schwangerschaftswoche mit einer feinen, retikulogranulären Eintrübung beider Lungen (Atemnotsyndrom, ANS, Grad I). b Röntgenbild eines Frühgeborenen der 31. Schwangerschaftswoche mit einer feingranulären, pulmonalen, bilateralen Verdichtung und einem zusätzlichen positiven Bronchopneumogramm, das die Herzgrenzen überschreitet; die Herz- und Zwerchfellkonturen sind noch abgrenzbar (ANSGrad II). cUnmittelbarnach endotrachealer Surfactantgabezeigt sich eine deutliche Regredienz der Röntgenbefunde bei dem Frühgeborenen der 31. Schwangerschaftswoche. Nebenbefundlich ist auf allen 3 Aufnahmen eine Magensonde abgebildet, in b zudem ein Einschwemmkatheter via linken Arm mit Projektion der Spitze auf den rechten Vorhof. In c wurde der Einschwemmkatheter zurückgezogen mit nun Spitzenprojektion auf den Übergang der V. cava superior zum rechten Vorhof, zudem erfolgte eine Intubation zur Applikation des Surfactants
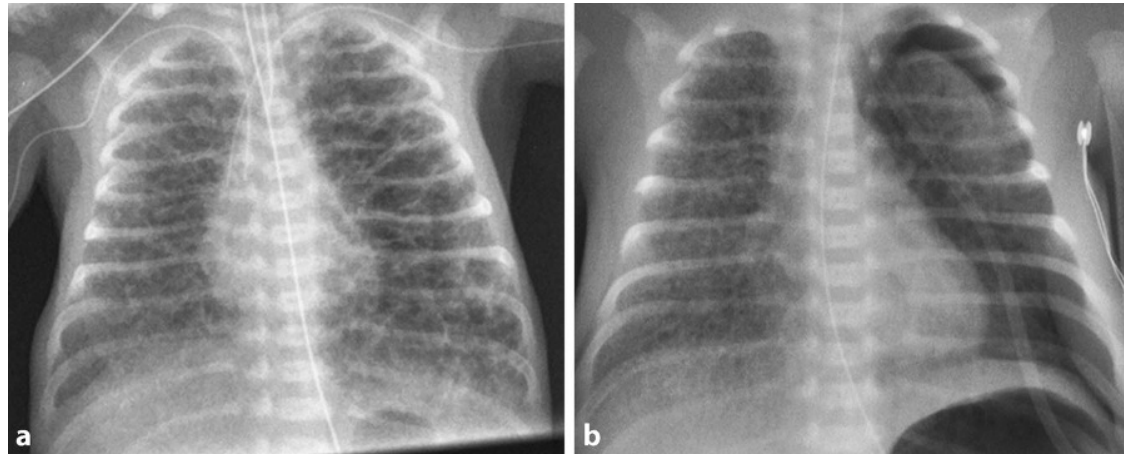

Abb. $2 \Delta$ a Röntgenbild eines pulmonal-interstitiellen Emphysems (PIE) mit ubiquitären zystoiden Aufhellungen beidseits und Emphysemzeichen mit tief stehenden Zwerchfellschenkeln und schlankem Herz- und Gefäßdiameter. Nebenbefundlich sind Einschwemmkatheter via beide Arme mit Projektionen der Katheterspitzen auf die V. cava superior sowie eine korrekte Tubus- und Magensondenlage abgebildet. b Die zweite Röntgenaufnahme zeigt die Komplikation eines PIEmit einem Mantelpneumothorax links; mit abgebildet Magensonde

30 Jahren um den Faktor 2 bis 3 reduziert werden. Nach Angaben von Huda [3] berechnet sich die effektive Dosis einer Röntgen-Thorax-Aufnahme im p.-a.-Strahlengang für ein 5-jähriges Kind mit etwa $0,007 \mathrm{mSv}$ und für ein 10-jähriges Kind mit etwa 0,011 mSv. Die vom Bundesamt für Strahlenschutz veröffentlichten Dosisreferenzwerte aus dem Jahr 2016 [4] können bei korrekter Durchführung der Röntgen-Thorax-Aufnahmen, inklusive richtiger Einblendung, ohne Schwierigkeiten unterschritten werden [5]. Aufgrund der höheren Strahlenempfindlichkeit von Kindern im Vergleich zu erwachsenen Patienten muss die Indikation für die Durchführung einer pädiatrischen Rönt- genuntersuchung des Thorax sorgfältig geprüft werden und auch strahlenfreie bilddiagnostische Alternativen, wie die Sonographie und die Magnetresonanztomographie (MRT), in Betracht gezogen werden. Der Fokus dieses Übersichtsartikels liegt auf den häufigen Indikationen für eine pädiatrische Röntgen-Thorax-Aufnahme, darunter gängigen Indikationen in der Neugeborenenperiode, der Frage nach entzündlichen Lungenveränderungen, der Aspiration von Fremdkörpern, der Detektion von Rundherden und der zystischen Fibrose.

\section{Indikationen in der Neonatal- periode}

Verglichen mit älteren Kindern erhalten Früh- und Neugeborene proportional häufiger eine Röntgenuntersuchung des Thorax. Eine typische Erkrankung von Frühgeborenen (Geburt vor Beendigung der 37. Schwangerschaftswoche) ist das Atemnotsyndrom (ANS), auch als Syndrom der hyalinen Membranen bezeichnet, auf Englisch "respiratory distress syndrome" (RDS). Klinisch benötigt das Kind in der Regel eine externe Sauerstoffzufuhr. Bei Frühgeborenen vor der 30. Schwangerschaftswoche tritt das ANS in ca. $30 \%$ der Fälle, bei Frühgeborenen vor der 28. Schwangerschaftswoche in ca. $90 \%$ der Fälle auf [6]. Auch Neugeborene können an einem ANS in ca. $1 \%$ der Fälle erkranken $[6,7]$. Die Hauptursache für das Auftreten eines ANS ist ein Mangel der oberflächenaktiven Substanz Surfactant. Die Synthese von Surfactant beginnt in der 24.-25. Schwangerschaftswoche in den Pneumozyten Typ Il; eine adäquate Produktion wird erst in der 36.-37. Schwangerschaftswoche erzielt [7]. Surfactant kleidet die Alveoleninnenfläche aus, senkt dabei die Oberflächenspannung der Alveolen und verhindert so ihren Kollaps während der Exspiration. Im Fall eines Surfactantmangels kommt es zu einem Alveolarkollaps mit der Entwicklung von Atelektasen, einer herabgesetzten Lun- 


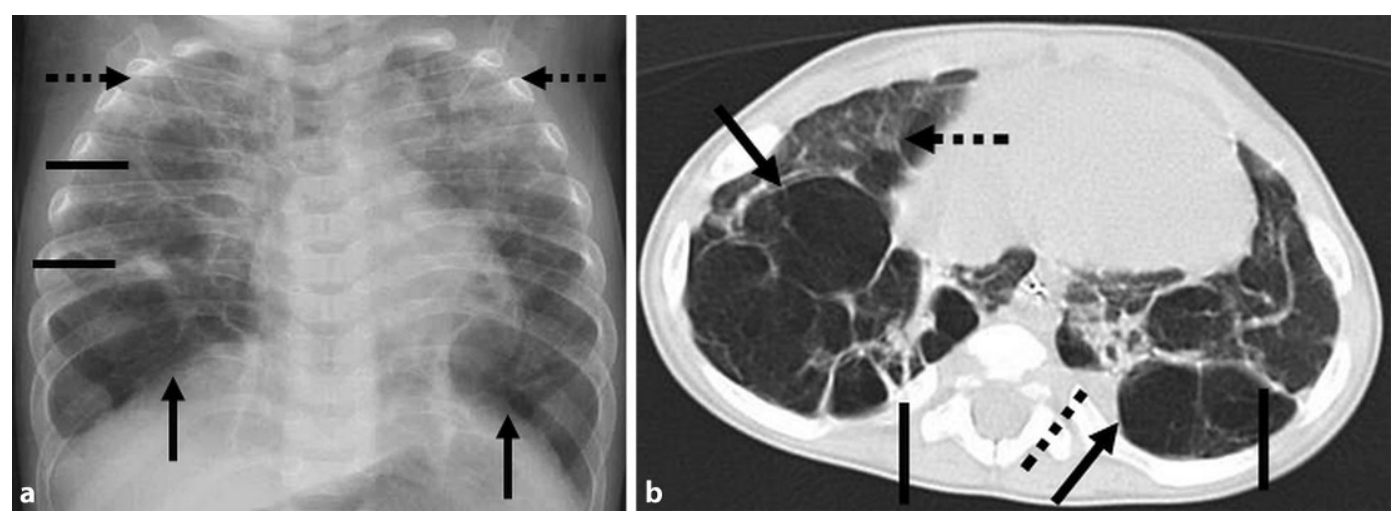

Abb. $3 \Delta$ a Die Röntgenaufnahme einer bronchopulmonalen Dysplasie zeigt ein Nebeneinander von zystischen Aufhellungen (durchgezogene Pfeile), flächigen milchglasartigen Verdichtungen (gestricheltePfeile) und streifig-narbigen Veränderungen (Linien). b Die Computertomographie (CT) durch die basalen Lungenabschnitte stellt die pulmonalen Manifestationen sensitiver dar: große Zysten (durchgezogene Pfeile), Milchglasareal (gestrichelter Pfeile), streifig-narbige Veränderungen (durchgezogene Linien) und ein Konsolidierungsareal im Lungensegment 10 links (gestrichelte Linie)

gencompliance und einem damit einhergehenden eingeschränkten Gasaustausch; die terminalen Bronchiolen und Alveolen sind mit Plasmatranssudationen, nekrotischen Zellen und Fibrin ausgekleidet, den sog. hyalinen Membranen [6, 8]. In der Übersichtsradiographie zeigen sich die ersten Zeichen für ein ANS oft unmittelbar nach der Geburt, wobei die maximale Ausprägung typischerweise erst $12-24 \mathrm{~h}$ nach der Geburt zur Abbildung kommt [9]. Anhand des Röntgenbilds wird das ANS in 4 Schwergrade eingeteilt $[6,9]$;

- Abb. 1:

- Grad I: Schlierige Lungeneintrübung mit einem retikulogranulären Muster

- Grad II: plus positives Bronchopneumogramm, das die Herzgrenzen überschreitet

- Grad III: plus Unschärfe der Herz-, Mediastinal- und Zwerchfellkonturen

- Grad IV: totale homogene Verschattung beider Lungen ohne abgrenzbaren Herz-/Mediastinalschatten (weiße Lunge)

Eine wesentliche Prophylaxe bzw. Therapie des ANS besteht in einer pränatalen Steroidapplikation der Mutter bei drohender Frühgeburt und einer frühzeitigen, postnatalen, intratrachealen Applikation von Surfactant.

Eine wichtige pulmonale Komplikation in der Neonatalperiode ist das pulmonalinterstitielle Emphysem (PIE), das wie das ANS vor allem bei Frühgeborenen auftritt. Das PIE entsteht aufgrund eines hohen transpulmonalen Drucks, der wegen ei- ner Überdehnung der Bronchioli terminalis und der Alveolen auftritt $[6,8,9]$. Kommt es zu einer Ruptur der überdehnten Alveolen und terminalen Bronchiolen, gelangt Luft in das Interstitium und die Lymphgefäße $[6,8,9]$. In der Röntgen-Thorax-Aufnahme stellt sich das PIE typischerweise mit zystoiden und linearen Aufhellungen mit je ca. $2 \mathrm{~mm}$ Durchmesser dar (ब Abb. 2); die Lokalisation ist häufig diffus und bilateral und reicht von perihilär nach peripher [6, $8,9]$. Nach Swischuk werden die bläschenartigen Aufhellungen eingeteilt in rundliche Bubbles (Typ I) und meander-wurmartige, tubuläre Bubbles (Typ II; [6]). Die zystoiden Aufhellungen können zu größeren Pseudozysten konfluieren. Weitere typische röntgenmorphologische Charakteristika sind Emphysemzeichen mit tief stehenden Zwerchfellschenkeln, schlankem Herz- und Mediastinumdurchmesser sowie verbreiterten Zwischenrippenräumen. Ursachen für ein PIE mit einer erhöhten Rupturgefährdung der Alveolen und terminalen Bronchiolen sind neben der Frühgeburtlichkeit eine langanhaltende mechanische Beatmung, eine Mekoniumaspiration oder eine perinatale Aspiration oder Pneumonie $[6,8,9]$. Komplikationen des PIE sind das Auftreten eines Pneumothorax (- Abb. 2), Pneumomediastinums, Pneumoperikards und Luftembolie $[6,8$, 9]. Häufig bildet sich das PIE ohne die Entwicklung von Spätmanifestationen und Langzeitkomplikationen zurück [8].

Im Gegensatz dazu persistiert die bronchopulmonale Dysplasie (BPD), auch als "chronic lung disease (CLD) of prema- turity" bezeichnet, und führt zu länger anhaltenden Komplikationen. Die klassische Form der BPD wurde im Jahr 1967 durch Northway et al. [10] als Folge einer langanhaltenden maschinellen Beatmung von Frühgeborenen beschrieben. Die chronologisch auftretenden pulmonalen Röntgenveränderungen wurden von Northway et al. in 4 Stadien eingeteilt und reichen von schweren ANS-Manifestationen bis zu grobzystischen, streifigen Lungenveränderungen $[6,8,10]$. Aufgrund einer Änderung des Therapieregimes durch eine pränatale Steroidgabe an die Mutter, endotracheale Surfactantapplikation und schonendere, moderne Beatmungstechniken mit geringeren Sauerstoffkonzentrationen tritt die klassische Form der BPD heute kaum noch auf $[6,11$, 12]. Die aktuellen klinischen Definitionen teilen die BPD anhand des Bedarfs an atemunterstützenden Maßnahmen und des Gestationsalters ein [12]. Die BPD ist die häufigste Form der infantilen chronischen Lungenerkrankungen und tritt bei ca. $10-25 \%$ der europäischen Kinder auf, die zwischen der 24. und $<32$. Schwangerschaftswoche geboren wurden [11]. Mögliche radiologische Manifestationen der BPD sind Konsolidierungen, zystische Veränderungen, Milchglasverdichtungen und angrenzende Überblähungsareale mit konsekutivem Mosaikmuster, Bronchialwandverdickungen und Fibrosezeichen, z.B. irreguläre Verdickung der interlobulären Septen [11, 13]. Wie die meisten interstitiellen Lungenerkrankungen im Kindesalter („,childhood interstitial lung 
Tab. 1 Typische Erreger ambulant erworbener pädiatrischer Pneumonien in Abhängigkeit vom Patientenalter. (Einteilung nach Ostapchuk et al. [14])

\begin{tabular}{|l|l|}
\hline Patientenalter & $\begin{array}{l}\text { Typische Erreger ambulant erworbener Pneumonien in dem jeweiligen } \\
\text { Patientenalter }\end{array}$ \\
\hline $\begin{array}{l}\text { Neugeborene } \\
\text { bis 20 Lebensta- } \\
\text { ge }\end{array}$ & $\begin{array}{l}\text { B-Streptokokken, Escherichia coli, Listeria monocytogenes, Streptococcus } \\
\text { pneumoniae, D-Streptokokken, Haemophilus influenzae, Ureaplasma } \\
\text { urealyticum, anaerobe Bakterien, Zytomegalievirus, Herpes-simplex-Virus }\end{array}$ \\
\hline $\begin{array}{l}3 \text { Lebenswo- } \\
\text { chen bis 3 Le- } \\
\text { bensmonate }\end{array}$ & $\begin{array}{l}\text { Streptococcus pneumoniae, Chlamydia trachomatis, Bordetella pertussis, } \\
\text { Haemophilus influenzae, Moraxella catarrhalis, Staphylococcus aureus, } \\
\text { Ureaplasma urealyticum, Virale Erreger (,respiratory syncytial virus“ [RSV], } \\
\text { Adeno-, Zytomegalie-, Influenza- und Parainfluenzaviren) }\end{array}$ \\
\hline $\begin{array}{l}\text { 3 Lebenswo- } \\
\text { chen bis 5 Le- } \\
\text { bensjahre }\end{array}$ & $\begin{array}{l}\text { Chlamydia pneumoniae, Mycoplasma pneumoniae, Streptococcus pneu- } \\
\text { moniae, Haemophilus influenzae, Moraxella catarrhalis, Staphylococcus } \\
\text { aureus, Mycobacterium tuberculosis, Neisseria meningitis, virale Erreger } \\
\text { (RSV, Rhino-, Adeno-, Influenza- und Parainfluenzaviren) }\end{array}$ \\
\hline $\begin{array}{l}\text { 5 Lebensjahre } \\
\text { bis Jugendalter }\end{array}$ & $\begin{array}{l}\text { Chlamydia pneumoniae, Mycoplasma pneumoniae, Streptococcus pneu- } \\
\text { moniae, Haemophilus influenzae, Legionellen, Staphylococcus aureus, } \\
\text { Mycobacterium tuberculosis, virale Erreger (RSV, Rhino-, Adeno-, Herpes-, } \\
\text { Influenza- und Parainfluenzaviren) }\end{array}$ \\
\hline
\end{tabular}

disease", CHILD) werden die pulmonalen Manifestationen detaillierter in der Computertomographie (CT) verglichen mit der Röntgenuntersuchung visualisiert (- Abb. 3). Auch der MRT mit der Möglichkeit der Darstellung von Pathologien in verschiedenen Wichtungen und der Durchführung funktioneller Ventilationsund Perfusionsaufnahmen ohne Strahlenexposition kommt eine zunehmende Bedeutung in der Diagnostik der BPD zu [11].

Weitere mögliche Indikationen für eine Röntgenuntersuchung des Thorax in der Neugeborenenperiode sind das Mekoniumaspirationssyndrom, die Diagnostik einer perinatalen Pneumonie und von Fehlbildungen, z. B. Ösophagusatresie, die kongenitale Zwerchfellhernie, die Lungensequestration und die "congenital pulmonary airway malformation“ (CPAM). Bei Vorliegen einer angeborenen Lungenfehlbildung ist eine sensitivere Schnittbilddiagnostik (CT/MRT) jedoch in der Regel indiziert, um etwa die pulmonale Gefäßsituation, wie beispielsweise bei einem Lungensequester, präoperativ abzubilden.

\section{Entzündliche Lungen- erkrankungen}

Eine der häufigsten Gründe für die Durchführung einer Röntgenuntersuchung des Thorax im Kindes- und Jugendalter ist die Frage nach entzündlichen Lungenerkrankungen. Welche Erreger eine Infektion der unteren Atemwege verursachen, hängt auch von dem Lebensalter der Kinder und Jugendlichen $a b[14,15]$. Postnatal können B-Streptokokken und gramnegative Darmbakterien eine Pneumonie verursachen, indem sie während des Geburtsvorgangs übertragen werden [14]. Anschließend ist bis zu einem Lebensalter von 3 Monaten Streptococcus pneumoniae der häufigste Erreger [14]. Bis zum Schulalter überwiegen virale Pneumonien mit „respiratory syncytial virus" (RSV) als dem häufigsten Keim, wohingegen im Schulalter bakterielle Infektionen mit Mycoplasma pneumoniae und Chlamydia pneumoniae dominieren [14]. In - Tab. 1 wird ein Überblick über typische Erreger von pädiatrischen Pneumonien in Abhängigkeit von dem Alter der Patienten gegeben.

Einigen Erregern werden typische radiologische Charakteristika zugeschrieben. Eine eindeutige Zuordnung zu einem spezifischen Erreger kindlicher Pneumonien ist anhand des Röntgenbildes jedoch nicht möglich [16]. Literaturangaben zufolge führt eine pulmonale Infektion mit RSV typischerweise zu folgenden radiologischen Veränderungen: Bronchialwandverdickungen, Überblähungsarealen, Atelektasen und einer bihilären Lymphadenopathie [15-17]. Im Röntgenbild des Thorax ist es schwierig bis unmöglich, beim Vorliegen von perihilären streifigen Verdichtungen zwischen Bronchialwandverdickungen im Rahmen einer Bronchitis/ Bronchiolitis und einer z.B. interstitiellen Entzündung zu unterscheiden; zudem ist der Übergang von einer Bronchitis/
Bronchiolitis in eine Pneumonie fließend. Virale Erreger können ein interstitielles radiologisches Pattern verursachen (auch als atypische Pneumonie bezeichnet), wobei sich die Entzündung im Lungeninterstitium abspielt [18]. Das Röntgenmuster einer interstitiellen pulmonalen Entzündung ist eine bilaterale, perihilär betonte, streifige Zeichnungsvermehrung, oft begleitet von einer bihilären Lymphadenopathie (- Abb. 4a). Mycoplasma pneumoniae kann radiographisch zu einer retikulärstreifigen, interstitiellen Zeichnungsvermehrung führen, aber auch eine alveoläre Beteiligung mit konsekutiver flauer, milchglasartiger Verdichtung ist häufig; da die Unterlappen prädominant betroffen sind, kann ein begleitender, oft gering ausgeprägter Pleuraerguss auftreten ( $\mathbf{0}$ Abb. $\mathbf{4 b}$ ).

Die meisten bakteriellen Erreger rufen eine Entzündungsreaktion primär in den Alveolarräumen hervor, mit den folgenden drei typischen radiologischen Manifestationsformen [18]:

- Lobärpneumonie: Konsolidierung eines Lungenlappens, teils auch nicht des gesamten Lappens, sondern eines oder mehrerer Segmente eines Lappens (- Abb. 4c)

- Bronchopneumonie: Entzündung des flächig-fleckig dichteangehobenen, peribronchialen Lungenparenchyms über eine bronchogene Erregerverteilung

- Herdpneumonie: lokaler, herdförmiger (gelegentlich rundlich konfigurierter), konsolidierender Entzündungsfokus

Nationalen und internationalen Leitlinien zufolge sollte eine Röntgenuntersuchung des Thorax bei der Diagnostik einer ambulant erworbenen Pneumonie im Kindesalter nicht in jedem Fall eingesetzt werden, da der Einfluss auf das Patienten-Outcome nicht evident ist $[19,20]$. In der prospektiven, randomisierten Studie von Swingler et al. [21] betrug die mediane Genesungszeit 7 Tage in der Gruppe von Kindern mit einer ambulant erworbenen Infektion der unteren Atemwege, die eine Röntgendiagnostik des Thorax erhalten hatten, und war dabei identisch in der pädiatrischen Kohorte mit einer vergleichbaren Infektion, aber ohne Röntgendiagnostik. Die AWMF-Leitlinie „Management der ambulant erworbenen Pneumonie bei Kindern und Jugend- 

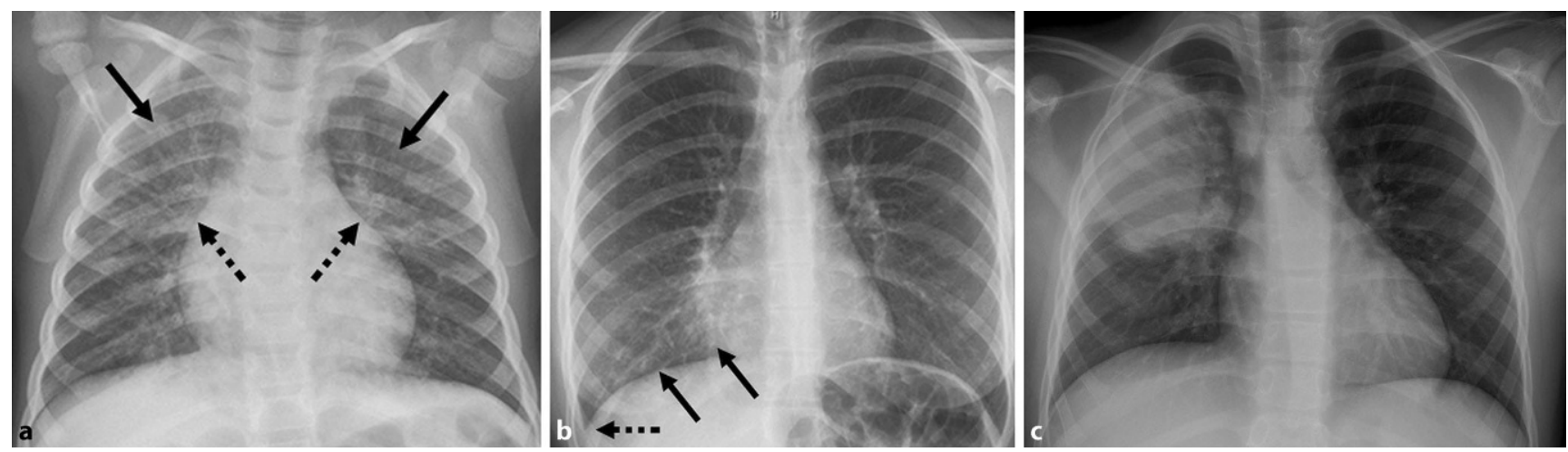

Abb. $4 \Delta$ a Röntgenmuster einer interstitiellen Viruspneumonie (hervorgerufen durch ein Influenzavirus) mit einer annähernd symmetrischen, bilateralen, perihilär betonten streifigen Zeichnungsvermehrung (durchgezogene Pfeile) und einer bihilären Lymphadenopathie (gestrichelte Pfeile). b Radiographisches Bild einer Pneumonie durch Mycoplasma pneumoniae mit einer retikulär-streifigen Zeichnungsvermehrung rechts basal, umgeben von einer flauen, milchglasartigen Verdichtung (durchgezogene Pfeile), und einem begleitenden rechten Randwinkelerguss (gestrichelter Pfeil). c Röntgenaufnahme einer bakteriellen Lobärpneumonie mit einem Konsolidierungsareal im rechten Oberlappen

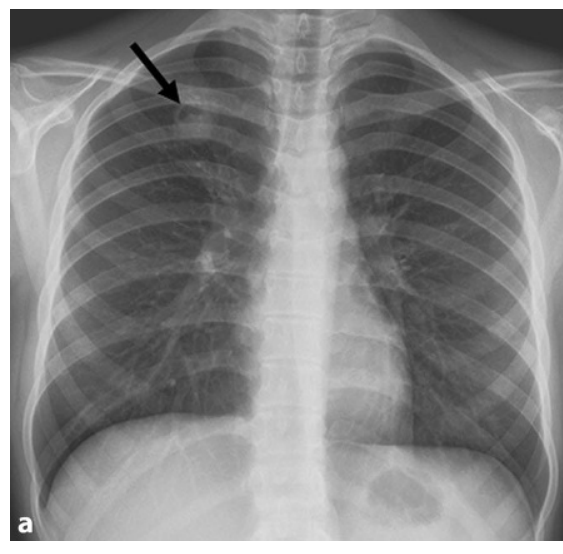

lichen (pädiatrische ambulant erworbene Pneumonie, p(AP)“ kommt sogar zu dem Statement, dass die Röntgenuntersuchung der Lunge bei Patienten mit nicht schwerer pCAP in der Regel verzichtbar ist, da radiologische Befundmuster nicht zuverlässig einer Ätiologie zugeordnet werden können [19]. Bei schweren Verläufen, z. B. einer nekrotisierenden Pneumonie, einem Lungenabszess, ist eine Röntgenaufnahme in jedem Fall indiziert, häufig gefolgt von einer Schnittbilddiagnostik (CT/MRT). Auch die Sonographie hat bei der Diagnostik entzündlicher Lungenerkrankungen einen wichtigen Stellenwert, da sie pleuranahe Konsolidierungen erkennen und die Qualität eines Pleuraergusses (serös-echofrei versus echoreich-septiert) zuverlässig beurteilen kann [19, 20].

Pilzinfektionen der Lunge treten vor allem bei immunsupprimierten Patienten auf, im Kindesalter z.B. nach einer Knochenmarktransplantation $[15,22]$.

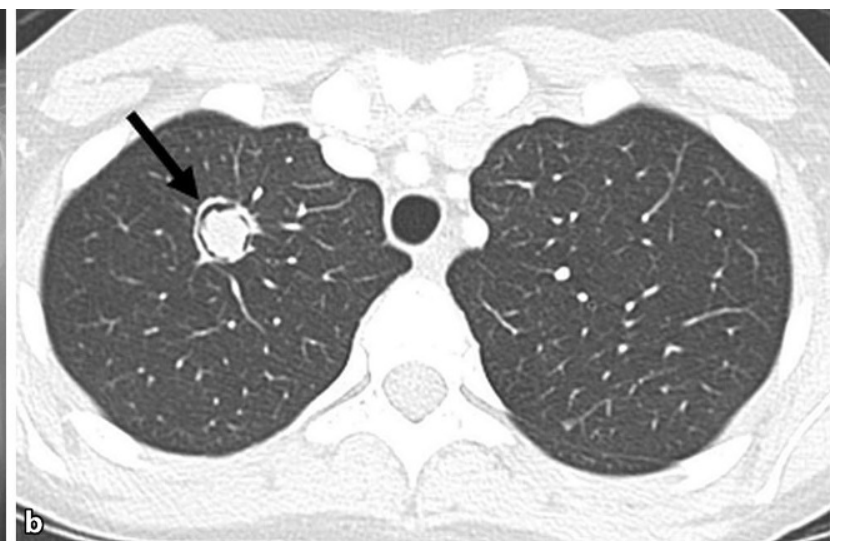

Abb. $5 \triangleleft$ Röntgenaufnahme (a) und Computertomographie (b) einer 16-jährigen Patientin mit einem Zustand nach Knochenmarktransplantation bei Rezidiv einer akuten myeloischen Leukämie und einerpulmonalen Aspergillose. Der länger bestehende Aspergillomherd im rechten Oberlappen weist ein peripheres Sichelzeichen auf (,, air crescent sign",Pfeil)
Dabei führt die pulmonale Aspergillose (zumeist verursacht durch Aspergillus fumigatus) typischerweise zu nodulären, dichten Veränderungen mit umgebender milchglasartiger Dichteanhebung (,HaloZeichen") [15, 22]. Im späteren Verlauf können die Nodi einschmelzen und eine Luftsichel aufweisen („air crescent sign“); - Abb. 5. Die Tuberkulose (zumeist verursacht durch Mycobacterium tuberculosis) kann bei Kindern jeglichen Alters auftreten, hat die höchste Inzidenz jedoch unter 5 Lebensjahren [15]. Das radiologische Leitsymptom der pulmonalen Tuberkulose im Kindesalter ist eine hiläre, teils auch paratracheale Lymphadenopathie (uni- oder bilateral lokalisiert), wobei eine Atelektase oder eine Überblähung eines Lungenabschnitts infolge einer Kompression der Trachea und/oder von Bronchien durch die vergrößerten Lymphknoten entstehen kann [15, 23]. Der pulmonale Primärkomplex besteht aus einem infiltra- tiven pulmonalen Herdbefund mit einer dazu gehörigen Lymphadenopathie, wobei zwischen den beiden eine streifige Lymphangitis vorliegen kann [15, 23]. Ein begleitender Pleuraerguss tritt seltener bei Kleinkindern, vor allem bei größeren Kindern und Adoleszenten auf [23]. Die wichtigste bildgebende Modalität bei der Diagnostik der pulmonalen Tuberkulose ist die Röntgenuntersuchung des Thorax [15]. SARS-CoV-2 („,severe acute respiratory syndrome coronavirus type $2^{\prime \prime}$ ) kann auch bei Kindern zu einer COVID-19Pneumonie mit ähnlichen pulmonalen Manifestationen wie bei erwachsenen Patienten führen, allerdings weisen Kinder im Vergleich zu erwachsenen Patienten häufiger Bronchialwandverdickungen auf [24]. 


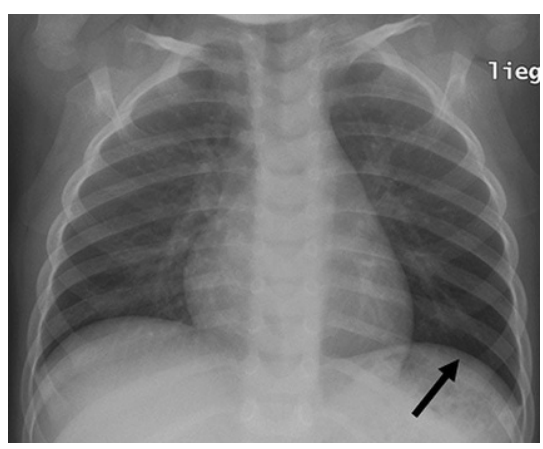

Abb. $6 \Delta$ Röntgenbild eines 23 Monate alten Jungen mit Zustand nach Aspiration einer Cashewnuss. Aufgrund einer inkompletten Atemwegsverlegung zeigt sich eine Überblähung der linken Lunge, vor allem basal (Pfeil). Bronchoskopisch konnten mehrere Anteile der Cashewnuss aus dem linken Hauptbronchus geborgen werden

\section{Fremdkörperaspiration}

Die Aspiration eines Fremdkörpers kann in allen Altersgruppen vorkommen, am häufigsten jedoch zwischen 12 Lebensmonaten und 3 Lebensjahren [25]. Eine Fremdkörperaspiration ist eine potenziell lebensbedrohliche Situation, die eine dringliche Diagnostik und Therapie erfordert. Daher sollte bei V.a. eine Fremdkörperaspiration eine zügige Bronchoskopie durchgeführt werden. In der klinischen Routine wird häufig eine Röntgenuntersuchung durchgeführt; maximal ein Viertel der aspirierten Fremdkörper sind jedoch röntgendicht [26]. Die häufigsten Fremdkörper sind Nüsse und Kerne, gefolgt von einer großen Bandbreite, wie z. B. Münzen oder Spielzeugteile [25, 26]. Aspirierte Nahrung kann Wasser aufnehmen und dadurch aufquellen sowie eine lokale Entzündungsreaktion hervorrufen, was die Atemwegsenge verstärkt. Das Röntgenbild kann unauffällig sein, eine pulmonale Atelektase und/oder Überblähung aufweisen, in Abhängigkeit auch von Art und Lokalisation des Fremdkörpers [15, 27]. Die häufigsten Fremdkörperlokalisationen sind die Haupt- und Segmentbronchien [15]. Bei einem kompletten Verschluss der Bronchien tritt eine Atelektase und ggf. eine konsekutive Pneumonie in den abhängigen Lungenarealen auf [15, 27]. Bei einem inkompletten Bronchusverschluss kann es zu einem Ventilmechanismus und einem konsekutiven obstruktiven Emphysem (Überblähung des betroffenen
Lungenabschnitts) kommen (• Abb. 6), wobei die Luft während der Inspiration in die Lunge gelangt, aber während der Exspiration nicht mehr entweichen kann [15, 27]. Diese Überblähung kann vor allem in Exspirationsaufnahmen beobachtet werden; in Durchleuchtungsaufnahmen können sich ein Mediastinalpendeln (mit Medistinalverlagerung in Exspiration zur gesunden Seite) und eine paradoxe Zwerchfellbeweglichkeit der betroffenen Lungenhälfte darstellen. Zusätzliche Bilddiagnostik soll jedoch die erforderliche Bronchoskopie wegen ihrer gleichzeitigen therapeutischen Möglichkeiten nicht verzögern. Ein unauffälliger radiologischer Befund schließt eine Fremdkörperaspiration nicht aus, hier muss bei klinischem Verdacht trotzdem bronchoskopiert werden. Die Ingestion von Knopfzellbatterien kann erhebliche Schäden an Ösophagus und/oder Trachea hervorrufen, wie z.B. Ulzerationen, Stenosen, Perforationen, Fisteln [28, 29]. Im Fall einer komplizierten Fremdkörperingestion kann eine CT-Untersuchung indiziert sein, etwa als native Low-dose-Untersuchung zur Detektion eines im Röntgenbild okkulten Fremdkörpers oder mit intravenöser Kontrastmittelapplikation zur präoperativen Planung.

\section{Detektion von pulmonalen Rundherden}

Einige bei Kindern und Jugendlichen typischerweise vorkommende Malignome metastasieren primär in die Lunge, darunter das Nephroblastom, Osteo- und EwingSarkom. Die CT-Untersuchung des Thorax ist die sensitivste bildgebende Modalität zur Detektion pulmonaler Rundherde und kann auch deutlich kleinere Rundherde $<3 \mathrm{~mm}$ im Vergleich zur Röntgenaufnahme darstellen [30]. Bei der Frage nach pulmonalen Metastasen kann die $\mathrm{CT}$ in nativer Low-dose-Untersuchung durchgeführt werden. Im Rahmen der Primärdiagnostik und Therapie eines $\mathrm{Ne}$ phroblastoms, Osteo- und Ewing-Sarkoms wird eine CT des Thorax zur Detektion pulmonaler Metastasen in den aktuell geltenden AWMF-Leitlinien empfohlen [31-33]. In der Tumornachsorge wird der Röntgenuntersuchung des Thorax, ggf. ergänzt durch eine CT-Diagnostik, in den aktuell geltenden AWMF-Leitlinien der Vorzug gegeben [31-33]; - Abb. 7. Die MRT wird zunehmend als bildgebende Alternative zur Diagnostik pulmonaler Rundherde diskutiert; ein Benefit der MRT scheint eine mögliche bessere Differenzierung zwischen benignen und malignen Rundherden im Vergleich $z u$ anderen Modalitäten zu sein [34, 35].

\section{Zystische Fibrose}

Die zystische Fibrose ("cystic fibrosis", CF; Mukoviszidose) ist die häufigste autosomal-rezessiv vererbte Erkrankung in Europa. Aufgrund des seit 2016 geltenden bundesweiten Neugeborenenscreenings wird die Krankheit in der Mehrheit der Fälle bereits im Neugeborenenalter diagnostiziert. Die Morbidität und Mortalität der Patienten wird in der Regel durch die pulmonalen CF-Manifestationen bestimmt. Der Multiorgankrankheit CF liegen Gendefekte zugrunde, die das auf dem langen Arm des Chromosoms 7 lokalisierte CFTR(„cystic fibrosis transmembrane conductance regulator")-Gen betreffen; das Gen kodiert für einen Chloridkanal in Drüsenzellmembranen [36]. Aufgrund eines Defektes des Chloridkanals entsteht ein zähflüssiges Sekret.

Pulmonal treten charakteristische CF-Veränderungen auf. Der zähflüssige Schleim stellt sich in Form einer Schleimretention ("mucus plugging“) dar und kann zu einer Verlegung der Atemwege (Mukoidimpaktion) führen [37, 38]. Auch als Folge von Entzündungsreaktionen können Bronchiektasen und Bronchialwandverdickungen sowie langfristig Konsolidierungen und Parenchymdestruktionen entstehen [37, 38]. Aufgrund von Überblähungen können sich ein Lungenemphysem sowie Bullae ausbilden, die bei subpleuraler Lokalisation durch ein ZerreiBen ihrer Wände zu einem Pneumothorax führen können [38]. Die beschriebenen pulmonalen CF-Manifestationen führen im Röntgenbild typischerweise zu einer streifigen Strukturvermehrung (• Abb. 8), Ring- und Fleckschatten, großflächigen Verdichtungen und Emphysemzeichen. Die charakteristischen Röntgenveränderungen wurden von Chrispin und Norman [39] im Jahr 1974 zu einem Score zusammengefasst, der im Wesentlichen bis 

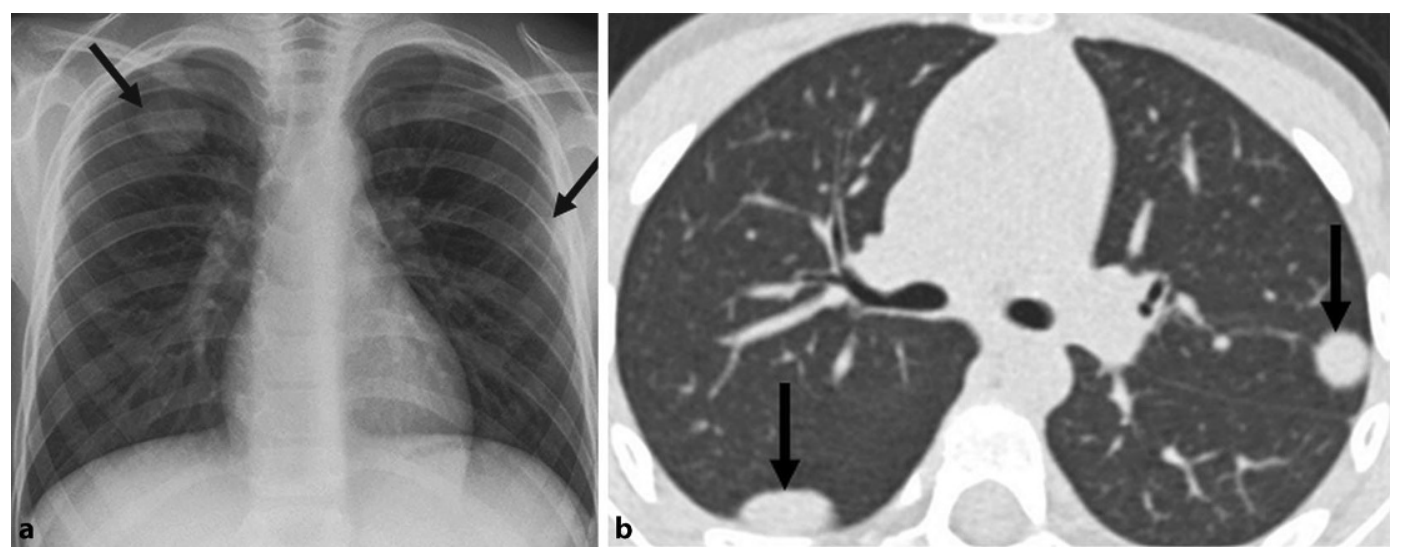

Abb. $7 \triangleleft$ Röntgenaufnahme (a) und Computertomographie $(\mathrm{CT}, \mathbf{b})$ eines 6-jährigen Jungen mit einem Zustand nach Nephroblastom. In der Tumornachsorge fand sich radiographisch (a) ein großer Rundherd im rechten und linken Oberlappen (Pfeile). Die $\mathrm{CT}$ (in nativer Low-doseTechnik) bestätigte die pulmonalen Filiae (Pfeile) und detektierte in dem Fall keine weiteren Rundherde

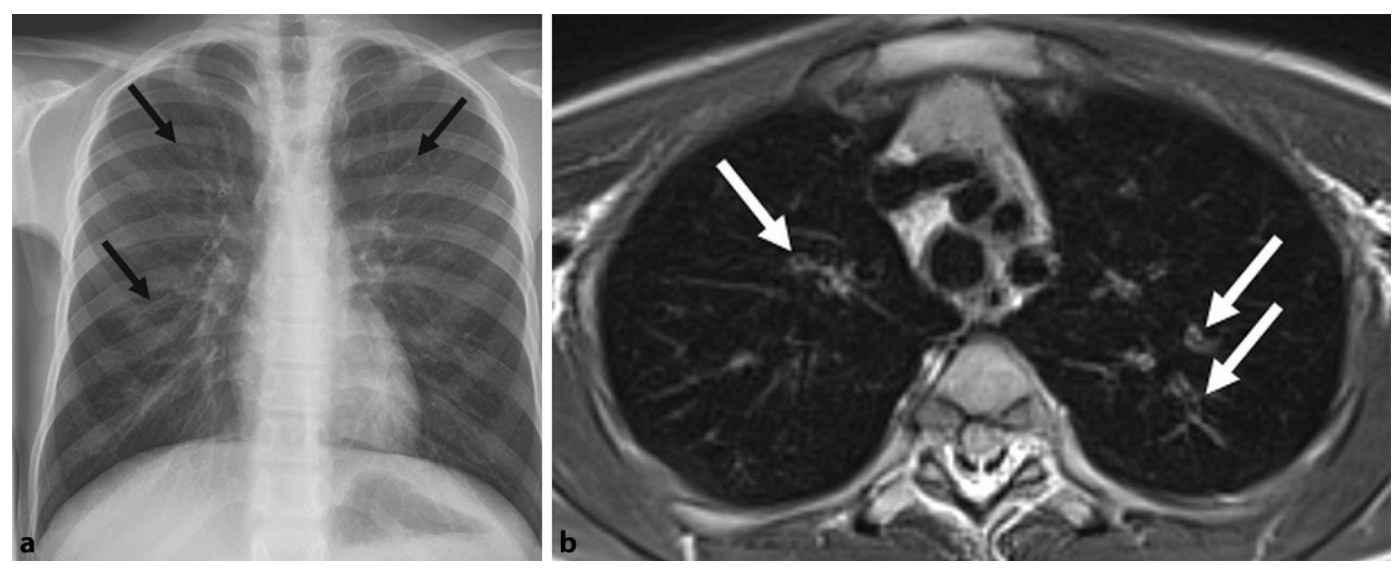

Abb. $8 \Delta$ a Das Röntgenbild eines 12-jährigen männlichen Patienten mit Mukoviszidose zeigt zentral betont eine geringe streifige Zeichnungsvermehrung und zarte Rundschatten als Hinweis für Bronchiektasen und Bronchialwandverdickungen (Pfeile). b In der anschließenden Magnetresonanztomographie (axiale T2-Wichtung) stellen sich die Bronchialwandverdickungen und Bronchiektasen in den Oberlappen deutlicher dar (Pfeile)

heute Gültigkeit hat. Dieser CN-Score setzt zwingend eine seitliche Röntgenaufnahme voraus, die generell bei Kindern und Jugendlichen aus Strahlenschutzgründen nur selten durchgeführt wird. Im Jahr 2005 entwickelten Benden et al. [40] einen modifizierten $\mathrm{CN}$-Score, bei dem nur eine Röntgenaufnahme in einer Ebene (im anteroposterioren bzw. posteroanterioren Strahlengang, je nach Patientenalter und Krankheitsschwere) erforderlich ist.

Generell wird eine jährliche Röntgenuntersuchung des Thorax bei Patienten mit CF empfohlen, bei klinischer Verschlechterung häufiger [37]. Die CT kann die charakteristischen pulmonalen Veränderungen sensitiver als die Röntgenuntersuchung abbilden und kann in der Regel in Low-dose-Technik durchgeführt werden [38]. Zunehmend etabliert sich die MRT als strahlenfreie Alternative (- Abb. 8), wobei ein wesentlicher Benefit der MRT ist, dass morphologische als auch funktionelle Parameter ohne jegliche Strahlenexposition akquiriert werden können [38, 41, 42]. Ein effektives MRTProtokoll bei CF beinhaltet T2- und T1gewichtete Sequenzen sowie eine Perfusionssequenz, die kontrastmittelgestützt durchgeführt werden kann [41, 43]. Perfusions- als auch Ventilationsaufnahmen können jedoch mittels der PREFUL(„,phase resolved functional lung ${ }^{\prime \prime}$ )-Technik auch ohne intravenöse Kontrastmittelapplikation und in freier Atmung akquiriert werden [44]. Aufgrund des großen Potenzials der MRT könnten nach der Übersichtsarbeit von Hirsch et al. [45] bis zu 90\% der pädiatrischen CT-Untersuchungen durch eine MRT ersetzt werden. Aufgrund der ubiquitären Verfügbarkeit und der schnellen und einfachen Durchführbarkeit wird die pädiatrische Röntgenuntersuchung des Thorax ihren wichtigen Stellenwert beibehalten, obwohl je nach klinischer Fragestellung alternative Bildgebungsmo- dalitäten in Erwägung gezogen werden müssen.

\section{Fazit für die Praxis}

- Die Röntgenaufnahme des Thorax ist die häufigste konventionelle Röntgenuntersuchung im Kindes- und Jugendalter; ihr hoher Stellenwert beruht auf der ubiquitären Verfügbarkeit und der schnellen und einfachen Durchführbarkeit.

- Durch verbesserte Technologien nimmt die Strahlenexposition von Röntgen-Thorax-Untersuchungen kontinuierlich ab.

- Die proportional häufigsten RöntgenThorax-Aufnahmen im Kindesalter werden in der Neugeborenenperiode akquiriert.

- Nach der Neonatalperiode sind typische Fragestellungen für eine Röntgenuntersuchung des Thorax im Kindesalter Fremdkörperaspiration, zystische Fibrose und entzündliche Lungenerkrankungen, wobei eine Röntgenaufnahme bei der Diagnostik einer ambulant erworbenen Pneumonie nicht in jedem Fall durchgeführt werden sollte. 
- In Abhängigkeit von der klinischen Fragestellung sollen alternative Bildgebungsmodalitäten (CT, MRT, Sonographie) in Betracht gezogen werden.

\section{Korrespondenzadresse}

\section{Prof. Dr. Dipl. journ. Diane Miriam Renz}

Institut für Diagnostische und Interventionelle Radiologie, Arbeitsbereich Kinderradiologie, Medizinische Hochschule Hannover Carl-Neuberg-Str. 1, 30625 Hannover, Deutschland renz.diane@mh-hannover.de

\section{Einhaltung ethischer Richtlinien}

Interessenkonflikt. D.M. Renz, C. Huisinga, A. Pfeil, J. Böttcher, N. Schwerk, F. Streitparth und J.Weidemann geben an, dass kein Interessenkonflikt besteht.

Für diesen Beitrag wurden von den Autoren keine Studien an Menschen oder Tieren durchgeführt. Für die aufgeführten Studien gelten die jeweils dort angegebenen ethischen Richtlinien.

\section{Literatur}

1. Seidenbusch MC, Schneider K (2009) Zur Strahlenexposition von Kindern in der pädiatrischen Radiologie. Teil 5: Organdosen bei der Röntgenuntersuchung des Thorax. Rofo 181:454-471

2. Seidenbusch MC, Schneider K (2008) Zur Strahlenexposition von Kindern in der pädiatrischen Radiologie. Teil 4: Einfalldosen bei der Röntgenuntersuchung des Thorax. Rofo 180:1082-1103

3. Huda W (2004) Assessment of the problem: pediatric doses in screen-film and digital radiography. Pediatr Radiol 34(Suppl 3):S173-S182

4. Bundesamt für Strahlenschutz (2016) Bekanntmachung der aktualisierten diagnostischen Referenzwerte für diagnostische und interventionelle Röntgenanwendungen (BAnz AT 15.07.2016 B8)

5. Schneider KO, Seidenbusch MC (2018) Technische Anforderungen. In: Riccabona M, Beer M, Mentzel HJ (Hrsg) Bildgebung des Thorax bei Neugeborenen und Kleinkindern. Springer, Berlin, S18-30

6. MentzelHJ, Beer M, Riccabona M (2018) Erkrankungen der Lunge. In: Riccabona M, Beer M, Mentzel HJ (Hrsg) Bildgebung des Thorax bei Neugeborenen und Kleinkindern. Springer, Berlin, S93-113

7. Edwards MO, Kotecha SJ, Kotecha S (2013) Respiratory distress of the term newborn infant. Paediatr Respir Rev 14:29-36

8. Liszewski MC, Stanescu AL, Phillips GS et al (2017) Respiratory distress in neonates:underlying causes and current imaging assessment. Radiol Clin North Am 55:629-644

9. Puig S, Hörmann M, Kuhle S et al (2000) Thoraxröntgen beim neonatologischen Patienten. Radiologe 40:43-51

10. Northway WH Jr, Rosan RC, Porter DY (1967) Pulmonary disease following respirator therapy of hyaline-membrane disease. Bronchopulmonary dysplasia. NEngl J Med 276:357-368

\section{Chest X-rays in children and adolescents. Indications and limitations}

Clinical issue: Chest X-ray is the most commonly performed X-ray examination in children and adolescents. The aim of this review is to present the benefit of this radiologic modality, but also its limitations.

Methods: Compared with older children, most X-ray examinations of the chest were performed in newborns. After the neonatal period, this review focusses on the diagnosis of inflammatory pulmonary changes, foreign body aspiration, detection of pulmonary nodules, and cystic fibrosis.

Methodological innovations: The radiation exposure of $X$-ray examinations is continuously decreasing due to technical innovations. However, other imaging modalities were also continuously being optimized; therefore, alternatives without radiation exposure, i.e., magnetic resonance imaging [MRI] and ultrasound, should be considered in case of specific clinical indications.

Practical recommendation: Even if the diagnostic performance of chest X-ray examinations is often minor compared to computed tomography or MRI, chest X-ray still has a high value in children and adolescents, due to its ubiquitous availability and the relatively simple acquisition.

\section{Keywords}

Neonatal period · Pneumonia - Cystic fibrosis - Computed tomography · Magnetic resonance imaging

11. Semple T, Akhtar MR, Owens CM (2017) Imaging bronchopulmonary dysplasia-a multimodality update. Front Med (Lausanne) 4:88

12. Gilfillan M, Bhandari A, Bhandari V (2021) Diagnosis and management of bronchopulmonary dysplasia. BMJ375:n1974

13. Semple TR, Ashworth MT, Owens CM (2017) Interstitial lung disease in children made easier ... well, almost. Radiographics 37:1679-1703

14. Ostapchuk M, Roberts DM, Haddy R (2004) Community-acquired pneumonia in infants and children. Am Fam Physician 70:899-908

15. Hahn G, Renz D (2018) Lungenerkrankungen jenseits des Neugeborenenalters. In: Riccabona M, Beer M, Mentzel HJ (Hrsg) Bildgebung des Thorax bei Neugeborenen und Kleinkindern. Springer, Berlin, S195-214

16. Schneider K (2018) Besonderheiten des RöntgenThorax im Kindesalter. Was Radiologen wissen sollten. Radiologe 58:359-376

17. Kern S, Uhl M, Berner R et al (2001) Respiratory syncytial virus infection of the lower respiratory tract: radiological findings in 108 children. Eur Radiol 11:2581-2584

18. Beigelman-Aubry C, Godet C, Caumes E (2012) Lung infections: the radiologist's perspective. Diagn Interv Imaging 93:431-440

19. Rose M, Liese J, Barker M et al (2017) S2kLeitlinie "Management der ambulant erworbenen Pneumonie bei Kindern und Jugendlichen (pädiatrische ambulant erworbene Pneumonie, pCAP)“(AWMF-Registernr. 048/013)

20. Andronikou S, Lambert E, Halton J et al (2017) Guidelines for the use of chest radiographs in community-acquired pneumonia in children and adolescents. Pediatr Radiol 47:1405-1411

21. Swingler GH, Hussey GD, Zwarenstein M (1998) Randomised controlled trial of clinical outcome after chest radiograph in ambulatory acute lower-respiratory infection in children. Lancet 351:404-408

22. Beyer F, Wormanns D (2011) Bildgebende Diagnostik von Pneumonien. Radiologe 51:405-418
23. Concepcion NDP, Laya BF, Andronikou $S$ et al (2017) Standardized radiographic interpretation of thoracic tuberculosis in children. Pediatr Radiol 47:1237-1248

24. Chen A, Huang JX, Liao Y et al (2020) Differences in clinical and imaging presentation of pediatric patients with COVID-19 in comparison with adults. Radiol Cardiothorac Imaging 2:e200117

25. Goyal S, Jain S, Rai G et al (2020) Clinical variables responsible for early and late diagnosis of foreign body aspiration in pediatrics age group. JCardiothorac Surg 15:271

26. Altuntas B, Aydın Y, Eroglu A (2016) Foreign bodies in trachea: a 25-years of experience. Eurasian JMed 48:119-123

27. Donnelly LF, Frush DP, Bisset GS 3rd (1998) The multiple presentations of foreign bodies in children. AJR Am J Roentgenol 170:471-477

28. Gerner P, Pallacks F, Laschat M et al (2019) Gesundheitsschäden nach Ingestion von Knopfzellbatterien im Kindesalter. Bundesgesundheitsblatt Gesundheitsforschung Gesundheitsschutz 62:1354-1361

29. Krom H, Visser M, Hulst JM et al (2018) Serious complications after button battery ingestion in children. Eur J Pediatr 177:1063-1070

30. Peuchot M, Libshitz HI (1987) Pulmonary metastatic disease: radiologic-surgical correlation. Radiology 164:719-722

31. Graf N, Furtwängler R, Schneider D et al (2016) S1Leitlinie „Nephroblastom (Wilms-Tumor)" (AWMFRegisternr. 025/004)

32. Hecker-Nolting $S$, Baumhoer D, Bielack $S$ et al (2021) S1-Leitlinie "Osteosarkome" (AWMFRegisternr. 025/005)

33. Dirksen U, Jürgens $H$, Eich HT et al (2014) S1-Leitlinie "Ewing-Sarkome des Kindes- und Jugendalters" (AWMF-Registernr. 025/006)

34. Renz DM, Herrmann KH, Kraemer M et al (2021) Ultrashort echo time MRI of the lung in children and adolescents: comparison with non-enhanced computed tomography and standard postcontrast T1w MRI sequences. Eur Radiol. https:// doi.org/10.1007/s00330-021-08236-7 
35. Schaefer JF, Vollmar J, Schick F et al (2004) Solitary pulmonary nodules: dynamic contrast-enhanced MR imaging - perfusion differences in malignant and benign lesions. Radiology 232:544-553

36. Rowe SM, Miller S, Sorscher EJ (2005) Cystic fibrosis. NEngl J Med 352:1992-2001

37. Ley-Zaporozhan J (2020) Thoraxbildgebung bei zystischer Fibrose: Radiographie. Radiologe 60:802-812

38. Renz DM, Dohna M, Böttcher J et al (2020) Magnetresonanztomographie der Lunge bei zystischer Fibrose. Monatsschr Kinderheilkd 168:406-415

39. Chrispin AR, Norman AP (1974) The systematic evaluation of the chest radiograph in cystic fibrosis. Pediatr Radiol 2:101-105

40. Benden C, Wallis C, Owens CM et al (2005) The Chrispin-Norman score in cystic fibrosis: doing away with the lateral view. Eur Respir J 26:894-897

41. Leutz-Schmidt $P$, Eichinger $M$, Sommerburg $O$ et al (2020) Magnetresonanztomographie der Lunge bei Mukoviszidose. Radiologe 60:813-822

42. Renz DM, Scholz O, Böttcher J et al (2015) Comparison between magnetic resonance imaging and computed tomography of the lung in patients with cystic fibrosis with regard to clinical, laboratory, and pulmonary functional parameters. Invest Radiol 50:733-742

43. Scholz O, Denecke T, Böttcher J et al (2017) MRI of cystic fibrosis lung manifestations: sequence evaluation and clinical outcome analysis. Clin Radiol 72:754-763

44. Behrendt L, Voskrebenzev A, Klimeš F et al (2020) Validation of automated perfusion-weighted phase-resolved functional lung (PREFUL)-MRI in patients with pulmonary diseases. J Magn Reson Imaging 52:103-114

45. Hirsch FW, Sorge I, Vogel-Claussen J et al (2020) The current status and further prospects for lung magnetic resonance imaging in pediatric radiology. Pediatr Radiol 50:734-749

\section{DEUTSCHER KREBSKONGRESS}

\section{JETZT ANMELDEN!}

W WW.DKK2022.DE // \#DKK 2022 